\title{
Impact of Open Access Publication Requirements on a Traditional, Society-Sponsored Geochemistry Journal and Potential Future Outcomes
}

\author{
JEFFREY G CATALANO
}

Geochimica et Cosmochimica Acta, Washington University in St. Louis

Presenting Author: catalano@wustl.edu

Subscription journals have long been a primary publisher of geochemistry research and face substantial impacts from the ongoing transition to open access publishing. Geochimica et Cosmochimica Acta (GCA) is a society-sponsored journal that is owned and published by Elsevier in a hybrid format. Only $\sim 10 \%$ of the articles published in GCA are open access with no growth since 2016, likely reflecting the substantial article publishing changes (APCs). An additional, major impact on GCA from the move towards open access has been the termination of subscription contracts between Elsevier and Projekt DEAL, the University of California system, and other entities. This has led many authors, reviewers, and associate editors from these communities to end their association with GCA. Despite these challenges, total submissions to GCA have increased $23 \%$ over the last five years, largely driven by an increasing number of manuscripts from China.

These and other impacts on GCA from the transition to open access publishing are outside of the journal's control but raise valid questions regarding its future sustainability. APCs for GCA (3250 USD/2850 EUR) are not viable expenses for most researchers; many other geochemistry journals have similar APCs. Attempts by Elsevier to create mirror, fully-open access journals simply circumvent the central problem: the cost of author-supported open access is not viable. Geochemists have little interest in or ability to pay APCs yet have no desire to place their work behind a paywall with increasingly limited access. Preprints are one mechanism for authors to increase access to their research, but deposition of accepted manuscript versions in repositories (i.e., green open access) is accompanied by a 24 month embargo period. A possible future outcome is the negotiation of contracts between institutions and publishers that include blanket open access fees for authors at the covered organizations. Widespread adoption of such agreements would provide near-complete open access without draining the limited research funds of authors. However, if a new access model is not forthcoming then the geochemistry community faces the prospect of eventually abandoning foundational journals like GCA in favor of alternative, low-cost or free open access venues. 